Proyecciones

Vol. 19, No 3, pp. 291-303, December 2000.

Universidad Católica del Norte

Antofagasta - Chile

\title{
ON THE APPROXIMATE SOLUTION OF IMPLICIT FUNCTIONS USING THE STEFFENSEN METHOD
}

\author{
IOANNIS K. ARGYROS \\ Cameron University, U. S. A. \\ EMIL CATINAS \\ and \\ ION PAVALOIU \\ Intitut de Calcul, Romania
}

\begin{abstract}
We use inexact Steffensen-Aitken-type methods to approximate implicit functions in a Banach space. Using a projection operator our equation reduces to solving a linear algebraic system of finite order. Semilocal convergence results as well as an error analysis are also provided.
\end{abstract}

AMS (MOS) Subject Classification: 65J15, 65B05, 47H17, $49 D 15$.

Key Words and Phrases: Steffensen-Aitken method, implicit function, projection operator. 


\section{Introduction}

Let $E, \Lambda$ be Banach spaces and denote by $U\left(x_{0}, R\right)$ the closed ball with center $x_{0} \in E$ and of radius $R \geq 0$. We will use the same symbol for the norm \|\| in both spaces. Let $P$ be a projection operator $\left(P=P^{2}\right)$ which projects $E$ on its subspace $E_{P}$ and set $Q=I-P$. Suppose that the nonlinear operators $F(x, \lambda)$ and $G(x, \lambda)$ with values in $E$ are defined for $x \in D$, where $D$ is some open convex subset of $E$ containing $U\left(x_{0}, R\right)$, and $\lambda \in U\left(\lambda_{0}, S\right)$ for some $\lambda_{0} \in \Lambda, S \geq 0$. For each fixed $\lambda \in U\left(\lambda_{0}, S\right)$ the operator $\operatorname{PF}(w, \lambda)$ will be assumed to be Fréchet-differentiable for all $w \in D$. Then $P F^{\prime}(x, \lambda)$ will denote the Fréchet-derivative of the operator $P F(w, \lambda)$ with respect to the argument $w$ at $w=x$. Moreover for each fixed $\lambda \in U\left(\lambda_{0}, S\right)$ the operator $P G(w, \lambda)$ will be assumed to be continuous for all $w \in D$.

In this study we are concerned with the problem of approximating a solution $x^{*}:=x^{*}(\lambda)$ of the equation

$$
F(x, \lambda)+G(x, \lambda)=0 .
$$

We introduce the inexact Steffensen-Aitken-type method

$$
\begin{gathered}
x_{n+1}(\lambda)=x_{n}(\lambda)-A\left(x_{n}(\lambda), \lambda\right)^{-1}\left(F\left(x_{n}(\lambda), \lambda\right)+G\left(x_{n}(\lambda), \lambda\right)\right) \\
-z\left(x_{n}(\lambda), \lambda\right) \quad(n \geq 0),
\end{gathered}
$$

where by $x_{0}$ we mean $x_{0}(\lambda)$. That is, $x_{0}$ depends on the $\lambda$ used in (2). $A(x, \lambda) \in L(E \times \Lambda, E)$ and is given by

$$
\begin{gathered}
A\left(x_{n}(\lambda), \lambda\right)=P\left[g^{1}\left(x_{n}(\lambda), \lambda\right), g^{2}\left(x_{n}(\lambda), \lambda\right) ; F\right]+P\left[g^{3}\left(x_{n}(\lambda), \lambda\right),\right. \\
\left.g^{4}\left(x_{n}(\lambda), \lambda\right) ; G\right] \quad(n \geq 0)
\end{gathered}
$$

where $[x(\lambda), y(\lambda) ; F]$ (or $[x(\lambda), y(\lambda) ; G])$ denotes divided difference of order one on $F$ (or $G$ ) at the points $x(\lambda), y(\lambda) \in D$, satisfying

$$
[x(\lambda), y(\lambda) ; F](y(\lambda)-x(\lambda))=F(y(\lambda), \lambda)-F(x(\lambda), \lambda)
$$

for all $x(\lambda) \neq y(\lambda), \lambda \in U\left(\lambda_{0}, S\right)$, and

$$
[x(\lambda), x(\lambda) ; F]=F^{\prime}(x(\lambda), \lambda), \quad \lambda \in U\left(\lambda_{0}, S\right)
$$


if $F(x(\lambda), \lambda)$ is Fréchet-differentiable at $x(\lambda)$ for all $\lambda \in U\left(\lambda_{0}, S\right)$. The operator $z: D \times U\left(\lambda_{0}, S\right) \rightarrow E$ is chosen so that iteration $\left\{x_{n}(\lambda)\right\}(n \geq$ 0 ) generated by (2) converges to $x^{*}$. The operator $g^{1}, g^{2}, g^{3}, g^{4}: D \times$ $U\left(\lambda_{0}, S\right) \rightarrow E$ are continuous.

The importance of studying inexact Steffensen-Aitken methods comes from the fact that many commonly used variants can be considered procedures of this type. Indeed approximation (2) characterizes any iterative process in which corrections are taken as approximate solutions of Steffensen-Aitken equations. Moreover we note that if for example an equation on the real line is solved $F\left(x_{n}(\lambda), \lambda\right)+$ $G\left(x_{n}(\lambda), \lambda\right) \geq 0$ and $A\left(x_{n}(\lambda), \lambda\right)$ overestimates the derivative $x_{n}-$ $A\left(x_{n}(\lambda), \lambda\right)^{-1}\left(F\left(x_{n}(\lambda), \lambda\right)+G\left(x_{n}(\lambda), \lambda\right)\right)$ is always "larger" than the corresponding Steffensen-Aitken iterate. In such cases a positive $z\left(x_{n}(\lambda), \lambda\right)(n \geq 0)$ correction term is appropriate.

It can easily be shown by induction on $n$ that under the above hypotheses $F\left(x_{n}(\lambda), \lambda\right)+G\left(x_{n}(\lambda), \lambda\right)$ belong to the domain of $A\left(x_{n}(\lambda), \lambda\right)^{-1}$ for all $n \geq 0$.

Therefore, if the inverses exist (as it will be shown later in the theorem), then the iterates $\left\{x_{n}(\lambda)\right\}$ can be computed for all $n \geq 0$. The iterates generated when $P=I$ (identity operator on $E$ ) cannot easily be computed in infinite dimensional spaces since the inverses may be too difficult or impossible to find. It is easy to see, however, that the solution of equations (2) reduces to solving certain operator equations in the space $E P$. If, moreover, $E P$ is a finite dimensional space of dimension $N$, we obtain a system of linear algebraic equations of at most order $N$. Special choices of the operators introduced above reduce our iteration (2) to earlier considered methods. Indeed we can have: for $g^{1}(x(\lambda), \lambda)=g^{2}(x(\lambda), \lambda)=x(\lambda), g^{3}(x(\lambda), \lambda)=g^{4}(x(\lambda), \lambda)=$ $0, z=0$ we obtain Newton methods considered in [3], [4], [5]; for $P=I$, no $\lambda, g^{1}(x)=g^{2}(x)=x(x \in D), g^{3}\left(x_{n}\right)=x_{n-1}(n \geq 1)$, $g^{4}\left(x_{n}\right)=x_{n}(n \geq 0), z_{n}=0(n \geq 0)$ we obtain Catinas method [4]; for $P=I$, no $\lambda, G(x)=0(x \in D), z_{n}=0(n \geq 0), g^{3}(x)=g^{4}(x)=0$, $g^{2}(x)=g^{1}(F(x))(x \in D)$, we obtain methods considered by Păvăloiu in [3], [4], [6], [7]. Our choices of the operators since they include all previous methods allow us to consider a wider class of problems.

We provide sufficient conditions for the convergence of iteration (2) to a locally unique solution $x^{*}(\lambda)$ of equation (1) as well as several 
error bounds on the distances $\left\|x_{n+1}(\lambda)-x_{n}(\lambda)\right\|$ and $\left\|x_{n}(\lambda)-x^{*}(\lambda)\right\|$ $(n \geq 0)$.

\section{Convergence Analysis}

We can now state and prove the following semilocal convergence result:

Theorem. Let F, G, P, Q be as in the introduction. Assume:

(a) there exist $x_{0}(\lambda) \in D, \lambda_{0} \in \Lambda$ such that $C:=C(\lambda)=A\left(x_{0}(\lambda), \lambda_{0}\right)$ is invertible. Set $B=C^{-1}$;

(b) there exist nonnegative numbers $a_{i}, R, S, i=1,2, \ldots, 15$ such that:

(7)

(8)

$$
\|B P([x, y ; F]-[v, w ; F])\| \leq a_{1}(\|x-v\|+\|y-w\|),
$$

$\left\|x-g^{1}(x, \lambda)\right\| \leq a_{2}\left\|A(x, \lambda)^{-1}(F(x, \lambda)+G(x, \lambda))-z(x, \lambda)\right\|$

$\left\|x-g^{2}(x, \lambda)\right\| \leq a_{3}\left\|A(x, \lambda)^{-1}(F(x, \lambda)+G(x, \lambda))-z(x, \lambda)\right\|$,

$\left\|g^{1}(x, \lambda)-g^{1}(y, \lambda)\right\| \leq a_{4}\|x-y\| \quad a_{4} \in[0,1)$,

$\| B\left(A\left(x_{n+1}, \lambda\right)\left(z\left(x_{n+1}, \lambda\right)\right)-A\left(x_{n}, \lambda\right)\left(z\left(x_{n}, \lambda\right)\right)\left\|\leq a_{7}\right\| x_{n+1}-x_{n} \|\right.$

$$
\left\|g^{2}(x, \lambda)-g^{2}(y, \lambda)\right\| \leq a_{5}\|x-y\| \quad a_{5} \in[0,1)
$$

$\left\|B P\left([x, y ; G]-\left[g^{3}(x, \lambda), g^{4}(x, \lambda) ; G\right]\right)\right\| \leq a_{8}\left(\left\|x-g^{3}(x, \lambda)\right\|+\right.$

$$
\left.\left\|y-g^{4}(x, \lambda)\right\|\right)
$$




$$
\left\|x-g^{4}(x, \lambda)\right\| \leq a_{10}\left\|A(x, \lambda)^{-1}(F(x, \lambda)+G(x, \lambda))-z(x, \lambda)\right\|
$$

$\| B\left(\left[g^{1}\left(x_{0}, \lambda\right), g^{2}\left(x_{0}, \lambda\right) ; F\right]-\left[g^{1}\left(x_{0}, \lambda_{0}\right), g^{2}\left(x_{0}, \lambda_{0} ; F\right]\right)\left\|\leq a_{14}\right\| \lambda-\lambda_{0} \|\right.$

and

$$
\begin{gathered}
\| B\left(\left[g^{3}\left(x_{0}, \lambda\right), g^{4}\left(x_{0}, \lambda\right) ; G\right]-\left[g^{3}\left(x_{0}, \lambda_{0}\right), g^{4}\left(x_{0}, \lambda_{0}\right) ; G\right]\right) \\
\left\|\leq a_{15}\right\| \lambda-\lambda_{0} \|
\end{gathered}
$$

for all $v, w, x, y \in U\left(x_{0}, R\right), \lambda \in U(\lambda, S)$;

(c) the sequence $\left\{z\left(x_{n}(\lambda), \lambda\right)\right\}(n \geq 0)$ is null for all $\lambda \in U\left(\lambda_{0}, S\right)$;

(d) for each fixed $\lambda \in U\left(\lambda_{0}, S\right)$ there exists a minimum nonnegative number $r^{*}:=r_{\lambda}^{*}$ satisfying

$$
T_{\lambda}\left(r^{*}\right) \leq r^{*} \quad \text { and } \quad r^{*} \leq R
$$

with $r:=r(\lambda)$,

$$
T_{\lambda}(r)=n+\frac{b_{1} r+b_{2}}{b(r)-b_{3} r} r
$$

where

$$
n:=n(\lambda) \geq\left\|x_{0}(\lambda)-x_{1}(\lambda)\right\|
$$

$b_{1}=a_{1}\left(1+a_{4}+a_{5}\right)+a_{8}\left(1+a_{11}+a_{12}\right), b_{2}=a_{6}+a_{13}+a_{7}$, $b_{3}=a_{1}\left(a_{2}+a_{3}\right)+a_{8}\left(a_{9}+a_{10}\right)$,

$b(r):=b(r, s)=1-\left(a_{1}\left(a_{4}+a_{5}\right)+a_{8}\left(a_{11}+a_{12}\right)\right) r-\left(a_{14}+a_{15}\right) S ;$ 
(e) $r^{*}, R, S$ also satisfy:

$$
\begin{gathered}
r^{*} \geq \max \left\{\frac{\left\|g^{1}\left(x_{0}(\lambda), \lambda\right)-x_{0}(\lambda)\right\|}{1-a_{4}}, \frac{\left\|g^{2}\left(x_{0}(\lambda), \lambda\right)-x_{0}(\lambda)\right\|}{1-a_{5}},\right. \\
\left.\| \frac{g^{3}\left(x_{0}(\lambda), \lambda\right)-x_{0}(\lambda) \|}{1-a_{11}, \frac{\left\|g^{4}\left(x_{0}(\lambda), \lambda\right)-x_{0}(\lambda)\right\|}{1-a_{12}}}\right\},(26)
\end{gathered}
$$

$$
c:=c(\lambda)=d(r, R)<1
$$

where

$$
d\left(e_{1}, e_{2}\right)=\frac{b_{1}\left(e_{1}+e_{2}\right)+b_{4}}{b\left(e_{1}\right)-b_{3}\left(e_{1}+e_{2}\right)}
$$

and

$$
b_{4}=a_{6}+a_{13}
$$

Then

(i) For each fixed $\lambda \in U\left(\lambda_{0}, S\right)$ the scalar sequence $\left\{t_{n}(\lambda)\right\}(n \geq 0)$ generated by

$$
t_{0}(\lambda)=0, \quad t_{1}(\lambda)=n
$$

$t_{n+1}(\lambda)=t_{n}(\lambda)+\frac{b_{1}\left(t_{n}(\lambda)-t_{n-1}(\lambda)\right)+b_{2}}{\alpha_{n} \beta_{n}}\left(t_{n}(1)-t_{n-1}(\lambda)\right) \quad(n \geq 1)$,

$$
\alpha_{n}:=\alpha_{n}(\lambda)=1-b_{3} \gamma_{n} \quad(n \geq 0)
$$

$\beta_{n}:=\beta_{n}(\lambda)=1-\left(a_{14}+a_{15}\right) S-\left[a_{1}\left(a_{4}+a_{5}\right)+a_{8}\left(a_{11}+a_{12}\right)\right] t_{n}(\lambda) \quad(n \geq 0)$, and

$$
\gamma_{n}:=\gamma_{n}(\lambda)=\left(t_{n}(\lambda)-t_{n-1}(\lambda)\right) \beta_{n}^{-1} \quad(n \geq 1)
$$


is monotonically increasing, bounded above by $r^{*}$ and $\lim _{n \rightarrow \infty} t_{n}(\lambda)=$ $r^{*}$;

(ii) the inexact Steffensen-Aitken method generated by (2) is well defined, remains in $U\left(x_{0}(\lambda), r^{*}\right)$ for all $n \geq 0$, and converges to a solution $x^{*}(\lambda) \in U\left(x_{0}(\lambda), r^{*}\right)$ of equation (1). Moreover if $z=0$ then $x^{*}(\lambda)$ is unique in $U\left(x_{0}(\lambda), R\right)$. Furthermore the following error bounds are true:

$$
\left\|x_{n+1}(\lambda)-x_{n}(\lambda)\right\| \leq \frac{b_{1}\left\|x_{n}(\lambda)-x_{n-1}(\lambda)\right\|+b_{2}}{\bar{\alpha}_{n} \bar{\beta}_{n}} \| x_{n}(\lambda)-x_{n-1}(\lambda)
$$

$$
\begin{gathered}
\| \quad(n \geq 1), \\
\left\|x_{n+1}(\lambda)-x_{n}(\lambda)\right\| \leq t_{n+1}(\lambda)-t_{n}(\lambda) \quad(n \geq 0), \\
\left\|x_{n}(\lambda)-x^{*}(\lambda)\right\| \leq r^{*}-t_{n}(\lambda) \quad(n \geq 0),
\end{gathered}
$$

where

$\bar{\beta}_{n}:=\bar{\beta}_{n}(\lambda)=1-\left(a_{14}+a_{15}\right)\left\|\lambda-\lambda_{0}\right\|-\left[a_{1}\left(a_{4}+a_{5}\right)+a_{8}\left(a_{11}+a_{12}\right)\right]$

$$
\left\|x_{n}(\lambda)-x_{0}(\lambda)\right\| \quad(n \geq 0)
$$

and

$$
\bar{\gamma}_{n}:=\bar{\gamma}_{n}(\lambda)=\left\|x_{n}(\lambda)-x_{n-1}(\lambda)\right\| \bar{\beta}_{n}^{-1} \quad(n \geq 1)
$$

Proof. (i) By (21) and (30) we deduce $0 \leq t_{0}(\lambda) \leq t_{1}(\lambda) \leq r^{*}$. Let us assume $0 \leq t_{k-1}(\lambda) \leq t_{k}(\lambda) \leq r^{*}$ for $k=1,2, \ldots, n$. Then it follows from (30) and (31) that $0 \leq t_{k}(\lambda) \leq t_{k+1}(\lambda)$. Hence, the sequence $\left\{t_{n}(\lambda)\right\}(n \geq 0)$ is monotonically increasing. Moreover by (31) and the induction hypotheses we get in turn

$$
\begin{aligned}
t_{k+1}(\lambda) & \leq t_{k}(\lambda)+\frac{b_{1} r^{*}+b_{2}}{b\left(r^{*}\right)-b_{3} r^{*}}\left(t_{k}(\lambda)-t_{k-1}(\lambda)\right) \\
& \leq \cdots \leq n+\frac{b_{1} r^{*}+b_{2}}{b\left(r^{*}\right)-b_{3} r^{*}} r^{*}=T_{\lambda}\left(r^{*}\right) \leq r^{*} \quad(\text { by }(21)) .
\end{aligned}
$$


That is the sequence $\left\{t_{n}(\lambda)\right\}(n \geq 0)$ is also bounded above by $r^{*}$. Since for each fixed $\lambda \in U\left(\lambda_{0}, S\right) r^{*}$ is the minimum nonnegative number satisfying (21) it follows that $\lim _{n \rightarrow \infty} t_{n}(\lambda)=r^{*}$.

(ii) By hypotheses (30), (23) and (22) it follows that $x_{1}(\lambda) \in$ $U\left(x_{0}(\lambda), r^{*}\right)$. Moreover from (26) we deduce $g^{1}\left(x_{0}(\lambda), \lambda\right), g^{2}\left(x_{0}(\lambda), \lambda\right)$, $g^{3}\left(x_{0}(\lambda), \lambda\right), g^{4}\left(x_{0}(\lambda), \lambda\right) \in U\left(x_{0}(\lambda), r^{*}\right)$. Let us assume $x_{k+1}(\lambda)$, $g^{1}\left(x_{k}(\lambda), \lambda\right), g^{2}\left(x_{k}(\lambda), \lambda\right), g^{3}\left(x_{k}(\lambda), \lambda\right), g^{4}\left(x_{k}(\lambda), \lambda\right) \in U\left(x_{0}(\lambda), r^{*}\right)$ for $k=0,1,2, \ldots, n$, and that (36) is true for $k=1,2, \ldots, n$ (since it is true for $k=0$ by (23) and (30)). Then from (9) and (26) we get

$$
\begin{aligned}
\left\|g^{1}\left(x_{k}(\lambda), \lambda\right)-x_{0}(\lambda)\right\| \leq & \left\|g^{1}\left(x_{k}(\lambda), \lambda\right)-g^{1}\left(x_{0}(\lambda), \lambda\right)\right\|+ \\
& \left\|g^{1}\left(x_{0}(\lambda), \lambda\right)-x_{0}(\lambda)\right\| \\
\leq & a_{4}\left\|x_{k}(\lambda)-x_{0}(\lambda)\right\|+ \\
& \left\|g_{1}^{1}\left(x_{0}(\lambda), \lambda\right)-x_{0}(\lambda)\right\| \leq r^{*} .
\end{aligned}
$$

That is $g^{1}\left(x_{n}(\lambda), \lambda\right) \in U\left(x_{0}(\lambda), r^{*}\right)$. Similarly we obtain $g^{2}\left(x_{n}(\lambda), \lambda\right)$, $g^{3}\left(x_{n}(\lambda), \lambda\right), g^{4}\left(x_{n}(\lambda), \lambda\right) \in U\left(x_{0}(\lambda), r^{*}\right)$. Using (6), (9), (10), (13), (16), (17), (19) and (20) we obtain

$$
\begin{aligned}
\| & B P\left(\left[g^{1}\left(x_{k}(\lambda), \lambda\right), g^{2}\left(x_{k}(\lambda), \lambda\right) ; F\right]+\right. \\
& {\left[g^{3}\left(x_{k}(\lambda), \lambda\right), g^{4}\left(x_{k}(\lambda), \lambda\right) ; G\right]-} \\
& {\left[g^{1}\left(x_{0}(\lambda), \lambda_{0}\right), g^{2}\left(x_{0}(\lambda), \lambda_{0}\right) ; F\right]-} \\
& g^{3}\left(x_{0}(\lambda), \lambda_{0}\right) g^{4}\left(x_{0}(\lambda), \lambda_{0}\right) ; G \| \\
\leq & \| B P\left(\left[g^{1}\left(x_{k}(\lambda), \lambda\right) ; g^{2}\left(x_{k}(\lambda), \lambda\right) ; F\right]-\right. \\
& {\left.\left[g^{1}\left(x_{0}(\lambda), \lambda_{0}\right), g^{2}\left(x_{0}(\lambda), \lambda_{0}\right) ; F\right]\right) \| } \\
+ & \| B P\left(\left[g^{3}\left(x_{k}(\lambda), \lambda\right), g^{4}\left(x_{k}(\lambda), \lambda\right) ; G\right]-\right. \\
& \left.\left.g^{3}\left(x_{0}(\lambda), \lambda_{0}\right), g^{4}\left(x_{0}(\lambda), \lambda_{0}\right) ; G\right)\right] \| \\
\leq & \| B P\left(\left[g^{1}\left(x_{k}(\lambda), \lambda\right), g^{2}\left(x_{k}(\lambda), \lambda\right) ; F\right]-\right. \\
& {\left[g^{1}\left(x_{0}(\lambda), \lambda\right), g^{2}\left(x_{0}(\lambda), \lambda\right)\right] \| } \\
+ & \| B P\left(\left[g^{1}\left(x_{0}(\lambda), \lambda\right), g^{2}\left(x_{0}(\lambda), \lambda\right) ; F\right]-\right. \\
& {\left.\left[g^{1}\left(x_{0}(\lambda), \lambda_{0}\right), g^{2}\left(x_{0}(\lambda), \lambda_{0}\right) ; F\right]\right) \| } \\
+ & \| B P\left(\left[g^{3}\left(x_{k}(\lambda), \lambda\right), g^{4}\left(x_{k}(\lambda), \lambda\right) ; G\right]-\right. \\
& {\left.\left[g^{3}\left(x_{k}(\lambda), \lambda\right), g^{4}\left(x_{0}(\lambda), \lambda\right)\right]\right) \| } \\
+ & \| B P\left(\left[g^{3}\left(x_{0}(\lambda), \lambda\right), g^{4}\left(x_{0}(\lambda), \lambda\right) ; G\right]-\right. \\
& {\left.\left[g^{3}\left(x_{0}(\lambda), \lambda_{0}\right), g^{4}\left(x_{0}(\lambda), \lambda_{0}\right) ; G\right]\right) \| } \\
\leq & a_{1}\left(a_{4}+a_{5}\right)\left\|x_{k}(\lambda)-x_{0}(\lambda)\right\|+a_{14}\left\|\lambda-\lambda_{0}\right\| \\
& +a_{8}\left(a_{11}+a_{12}\right)\left\|x_{k}(\lambda)-x_{0}(\lambda)\right\|+a_{15}\left\|\lambda-\lambda_{0}\right\| \\
\leq & {\left[a_{1}\left(a_{4}+a_{5}\right)+a_{8}\left(a_{11}+a_{12}\right)\right] r^{*}+\left(a_{14}+a_{15}\right) S<1 \text { by }(25) . }
\end{aligned}
$$


It follows from the Banach lemma on invertible operators [5] that $A\left(x_{k}(\lambda), \lambda\right)$ is invertible and

$$
\left\|A\left(x_{k}(\lambda), \lambda\right)^{-1} B^{-1}\right\| \leq \bar{\delta}_{k} \leq \delta_{k}
$$

where

$$
\bar{\gamma}_{k}=\left(\bar{\alpha}_{k} \bar{\beta}_{k}\right)^{-1} \quad \text { and } \quad \delta_{k}=\left(\alpha_{k} \beta_{k}\right)^{-1} \quad(k \geq 0) .
$$

Using (2) we obtain the approximation

$$
\begin{array}{r}
x_{k+1}(\lambda)-x_{k}(\lambda)=\left(A\left(x_{k}(\lambda), \lambda\right)^{-1} B^{-1}\right) B\left\{\left[P F\left(x_{k}(\lambda) \lambda\right)-\right.\right. \\
P F\left(x_{k-1}(\lambda), \lambda\right)-P\left[g^{1}\left(x_{k}(\lambda), \lambda\right), g^{2}\left(x_{k}(\lambda), \lambda\right) ; F\right] \\
\left.\left(x_{k}(\lambda)-x_{k-1}(\lambda)\right)\right]+\left[Q F\left(x_{k}(\lambda), \lambda\right)-Q F\left(x_{k-1}(\lambda), \lambda\right)\right]+ \\
{\left[A\left(x_{k}(\lambda), \lambda\right)\left(z\left(x_{k}(\lambda), \lambda\right)\right)-A\left(x_{k-1}(\lambda), \lambda\right)\left(z\left(x_{k-1}(\lambda), \lambda\right)\right)\right]+} \\
{\left[P G\left(x_{k}(\lambda), \lambda\right)-P G\left(x_{k-1}(\lambda), \lambda\right)-\right.} \\
\left.P\left[g^{3}\left(x_{k}(\lambda), \lambda\right), g^{4}\left(x_{k}(\lambda), \lambda\right) ; G\right]\left(x_{k}(\lambda)-x_{k-1}(\lambda)\right)\right]+ \\
\left.\left[Q G\left(x_{k}(\lambda), \lambda\right), G C\left(x_{k-1}(\lambda), \lambda\right)\right]\right\} \quad(k \geq 1) .
\end{array}
$$

By (6) we obtain

$$
\begin{aligned}
& \| B\left(P F\left(x_{k}(\lambda), \lambda\right)-P F\left(x_{k-1}(\lambda), \lambda\right)-\right. \\
& \left.P\left[g^{1}\left(x_{k}(\lambda), \lambda\right), g^{2}\left(x_{k}(\lambda)\right) ; F\right]\right)\left(x_{k}(\lambda)-x_{k-1}(\lambda)\right) \| \\
\leq & \| B P\left(\left[x_{k-1}(\lambda), x_{k}(\lambda) ; F-\right.\right. \\
& {\left.\left[g^{1}\left(x_{k-1}(\lambda), \lambda\right), g^{2}\left(x_{k-1}(\lambda), \lambda\right) ; F\right]\right)\|\| x_{k}(\lambda)-x_{k-1}(\lambda) \| } \\
\leq & a_{1}\left(\left\|x_{k-1}(\lambda)-g^{1}\left(x_{k-1}(\lambda), \lambda\right)\right\|+\left\|x_{k}(\lambda)-g^{2}\left(x_{k-1}(\lambda), \lambda\right)\right\|\right) \\
& \left\|x_{k}(\lambda)-x_{k-1}(\lambda)\right\| .
\end{aligned}
$$

Moreover from (7), (8), (9) and (10) we obtain the estimates

$$
\begin{aligned}
& \left\|x_{k-1}(\lambda)-g^{1}\left(x_{k-1}(\lambda), \lambda\right)\right\| \leq\left\|x_{k-1}(\lambda)-x_{k}(\lambda)\right\|+\left\|x_{k}(\lambda)-g^{1}\left(x_{k}(\lambda), \lambda\right)\right\| \\
& \leq\left\|g^{1}\left(x_{k}(\lambda), \lambda\right)-g^{1}\left(x_{k-1}(\lambda), \lambda\right)\right\| \\
& \leq\left\|x_{k}(\lambda)-x_{k-1}(\lambda)\right\|+a_{2}\left\|x_{k+1}(\lambda)-x_{k}(\lambda)\right\|+a_{4}\left\|x_{k}(\lambda)-x_{k-1}(\lambda)\right\|,
\end{aligned}
$$

$$
\begin{array}{r}
\left\|x_{k}(\lambda)-g^{2}\left(x_{k-1}(\lambda), \lambda\right)\right\| \leq\left\|x_{k}(\lambda)-g^{2}\left(x_{k}(\lambda), \lambda\right)\right\|+ \\
\left\|g^{2}\left(x_{k}(\lambda), \lambda\right)-g^{2}\left(x_{k-1}(\lambda), \lambda\right)\right\| \\
\leq a_{3}\left\|x_{k+1}(\lambda)-x_{k}(\lambda)\right\|+a_{5}\left\|x_{k}(\lambda)-x_{k-1}(\lambda)\right\|
\end{array}
$$


Hence from (43), (44) and (45) we get

$$
\begin{aligned}
& \| B P\left(F\left(x_{k}(\lambda), \lambda\right)-F\left(x_{k-1}(\lambda), \lambda\right)-\right. \\
& \left.\quad\left[g^{1}\left(x_{k}(\lambda), \lambda\right), g^{2}\left(x_{k}(\lambda), \lambda\right) ; F\right]\left(x_{k}(\lambda)-x_{k-1}(\lambda)\right)\right) \\
& \leq \quad a_{1}\left(1+a_{4}+a_{5}\right)\left\|x_{k}(\lambda)-x_{k-1}(\lambda)\right\|^{2}+ \\
& \quad a_{1}\left(a_{2}+a_{3}\right)\left\|x_{k+1}(\lambda)-x_{k}(\lambda)\right\|\left\|x_{k}(\lambda)-x_{k-1}(\lambda)\right\| .
\end{aligned}
$$

As in (46) but using (13), (14), (15), (16) and (17) we obtain

$$
\begin{aligned}
& \| B P\left(G\left(x_{k}(\lambda), \lambda\right)-G\left(x_{k-1}(\lambda), \lambda\right)-\right. \\
& \quad\left[g^{3}\left(x_{k}(\lambda), \lambda\right), g^{4}\left(x_{k}(\lambda), \lambda\right) ; G\right]\left(x_{k}(\lambda)-x_{k-1}(\lambda)\right) \| \\
& \leq a_{8}\left(1+a_{11}+a_{12}\right)\left\|x_{k}(\lambda)-x_{k-1}(\lambda)\right\|^{2}+ \\
& \quad a_{8}\left(a_{9}+a_{10}\right)\left\|x_{k+1}(\lambda)-x_{k}(\lambda)\right\|\left\|x_{k}(\lambda)-x_{k-1}(\lambda)\right\| .
\end{aligned}
$$

Furthermore from (11), (12) and (18) we get respectively

$$
\begin{gathered}
\left\|B\left(Q F\left(x_{k}(\lambda), \lambda\right)-Q F\left(x_{k-1}(\lambda), \lambda\right)\right)\right\| \leq a_{6}\left\|x_{k}(\lambda)-x_{k-1}(\lambda)\right\| \\
\| B\left(A\left(x_{k}(\lambda), \lambda\right)\left(z\left(x_{k}(\lambda), \lambda\right)\right)-A\left(x_{k-1}(\lambda), \lambda\right)\left(z\left(x_{k-1}(\lambda), \lambda\right)\right) \|\right. \\
\leq a_{7}\left\|x_{k}(\lambda)-x_{k-1}(\lambda)\right\|
\end{gathered}
$$

and

$$
\left\|B\left(Q G\left(x_{k}(\lambda), \lambda\right)-Q G\left(x_{k-1}(\lambda), \lambda\right)\right) \leq a_{13}\right\| x_{k}(\lambda)-x_{k-1}(\lambda) \| .
$$

Finally from (31), (41), (42), (46)-(50) we deduce that estimates (35) and (36) are true. By (36) and part (i) it follows that for each fixed $\lambda \in U\left(\lambda_{0}, S\right)$ iteration $\left\{x_{n}(\lambda)\right\}(n \geq 0)$ is Cauchy in a Banach space $E$ and as such it converges to some $x^{*}(\lambda) \in U\left(x_{0}(\lambda), r^{*}\right)$ (since $U\left(x_{0}(\lambda), r^{*}\right)$ is a closed set. Using hypothesis (c) and letting $n \rightarrow \infty$ in (2) we get $F\left(x^{*}(\lambda), \lambda\right)+G\left(x^{*}(\lambda), \lambda\right)=0$. That is $x^{*}(\lambda)$ is a solution of equation (1). Estimate (37) follows immediately from (36) by using standard majorization techniques [3], [5].

To show uniqueness when $z=0$, let us assume $y^{*}(\lambda) \in U\left(x_{0}(\lambda), R\right)$ is a solution of equation (1). Then from (2) we get 
$x_{n+1}(\lambda)-y^{*}(\lambda)=x_{n}(\lambda)-y^{*}(\lambda)-A\left(x_{n}(\lambda), \lambda\right)^{-1}\left[\left(F\left(x_{n}(\lambda), \lambda\right)\right.\right.$

$$
\left.\left.-F\left(y^{*}(\lambda), \lambda\right)\right)+\left(G\left(x_{n}(\lambda), \lambda\right)-G\left(y^{*}(\lambda), \lambda\right)\right)\right]
$$

Analyzing the right-hand side of (51) as in (42) with $y^{*}(\lambda)$ "replacing" $x_{k}(\lambda)$ and $x_{n}(\lambda)$ "replacing" $x_{k-1}(\lambda)$ we get

$\left\|x_{n+1}(\lambda)-y^{*}(\lambda)\right\| \leq c\left\|x_{n}(\lambda)-y^{*}(\lambda)\right\| \leq \cdots \leq c^{n+1}\left\|x_{0}(\lambda)-y^{*}(\lambda)\right\| \leq c^{n+1} R$.

By letting $n \rightarrow \infty$ in (52) and using (27) we get $\lim _{n \rightarrow \infty} x_{n+1}(\lambda)=$ $y^{*}(\lambda)$ for each fixed $\lambda \in U\left(\lambda_{0}, S\right)$. By the uniqueness of the limit of the sequence $\left\{x_{n}(\lambda)\right\}(n \geq 0)$ we deduce $x^{*}(\lambda)=y^{*}(\lambda)$.

That completes the proof of the Theorem.

Remarks. (1) Condition (6) implies that $F(x(\lambda), \lambda)$ is differentiable on $D$ [2], [3], whereas condition (13) does not necessarily imply the differentiability of $G(x(\lambda), \lambda)$ on $D$.

(2) Inequalities (21), (23), (25), (26) and (27) will determine $r^{*}, R$ and $S$.

(3) If $a_{2}+a_{4} \leq 1, a_{3}+a_{5} \leq 1, a_{9}+a_{11} \leq 1$ and $a_{10}+a_{12} \leq 1$ for $r^{*} \neq 0$, condition (26) is satisfied. Indeed from (7) we have

$$
\left\|g^{1}\left(x_{0}(\lambda), \lambda\right)-x_{0}(\lambda)\right\| \leq a_{2}\left\|x_{1}(\lambda)-x_{0}(\lambda)\right\| \leq a_{3} r^{*}
$$

and from (26) we must have

$$
\left\|g^{1}\left(x_{0}(\lambda), \lambda\right)-x_{0}(\lambda)\right\| \leq\left(1-a_{4}\right) r^{*}
$$

It suffices to show $a_{2} r^{*} \leq r^{*}\left(1-a_{4}\right)$ or $a_{2}+a_{4} \leq 1\left(r^{*} \neq 0\right)$ which is true by hypothesis. Similarly we can argue for the rest. 


\section{References}

[1] Argyros, I.K. On the convergence of some projection methods with perturbations, J. Comp. Appl. Math. 36, (1991), 255-258.

[2] Argyros, I.K. On an application of the Zincenko method to the approximation of implicit functions, Z.A.A. 10, 3, (1991), 391396.

[3] Argyros, I.K. and Szidarovszky, F. The Theory and Application of Iteration Methods, C.R.C. Press, Inc., Boca Raton, Florida, 1993.

[4] Cătinaş, E. On some iterative methods for solving nonlinear equations, Revue d'analyse Numerique et de theorie de l'approximation, 23, 1, (1994), 47-53.

[5] Kantorovich, L.V. The method of successive approximation for functional equations, Acta Math. 71 (1939), 63-97.

[6] Păvăloiu, I. Sur une generalisation de la methode de Steffensen, Revue d'analyse Numerique et de theorie de l'approximation, 21, 1, (1992), 59-65.

[7] Păvăloiu, I. Bilateral approximations for the solutions of scalar equations, Revue d'analyse numerique et de theorie de l'approximation, 23, 1, (1994), 95-100.

Received : July 1999.

Ioannis K. Argyros

Cameron University

Department of Mathematics

Lawton, OK 73505,

U.S.A.

e-mail : ioannisa@cameron.edu 


\section{Emil Cătinaş}

and

Ion Păvăloiu

Institul de Calcul

Str. Republicii Nr. 37

P. O. Box 68, 3400 Cluj-Napoca

Romania 